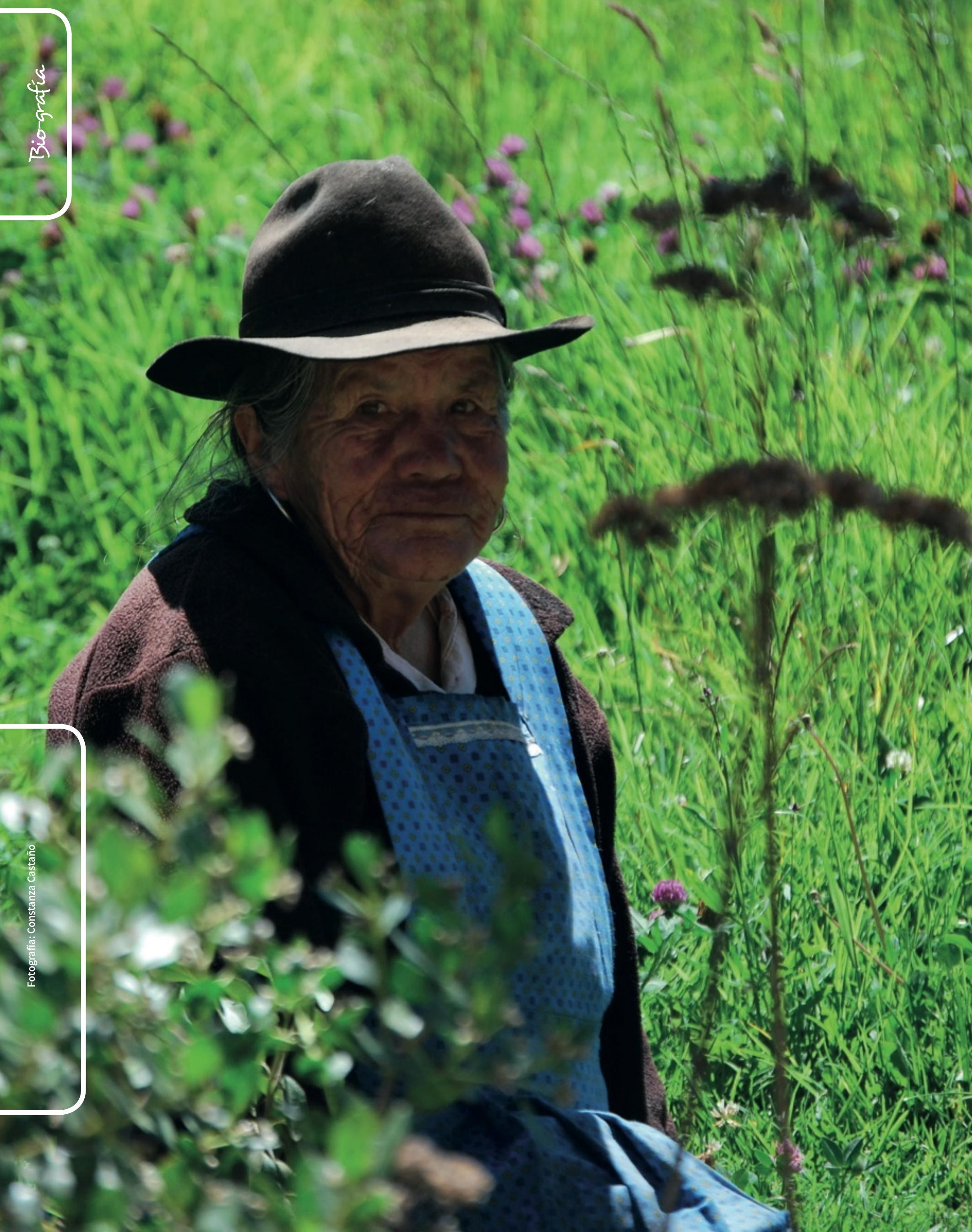




\title{
IDENTIDAD CULTURAL, REALIDAD BIOLÓGICA, ESPECIES DOMÉSTICAS Y APRENDIZAJE BASADO EN PROYECTOS: UNA PROPUESTA PARA LAS AULAS LATINOAMERICANAS
}

Fecha de recepción: 06 de agosto de 2013

Fecha de aprobación: 13 de diciembre de 2013

\author{
María del Rocío Téllez Estrada ${ }^{1}$ \\ Raúl Valadez Azúa²
}

\section{Resumen}

Actualmente, gran parte de los latinoamericanos estamos inmersos en un esquema donde vivimos por un lado la globalización y por otro tradiciones que son producto de siglos de historia en espacios biogeográficos específicos. En este sentido, los esquemas educativos tienen el mayor compromiso de salvaguardar nuestro acervo biológico (recursos) y cultural (tradiciones), para mantener en el educando la conciencia de que es parte de él. Dentro de los esquemas educativos actuales, en los que se promueve la enseñanza a través del desarrollo de habilidades y la interacción de las diversas disciplinas en pro de un pensamiento integrador y crítico, es fundamental disponer de elementos de estudio que permitan entender el fenómeno biológico, el histórico y el cultural de manera simultánea. Como propuesta a estas necesidades se plantea el uso de proyectos escolares dirigidos al estudio de animales, plantas y hongos domésticos latinoamericanos, dado que cada uno de ellos tiene una historia biológica y cultural integrada a las diversas regiones latinoamericanas; muchos han sido estudiados por diversas disciplinas y forman parte de la realidad de los habitantes de dichas regiones. Un caso ilustrativo aplicable a las aulas mexicanas es el perro pelón mexicano o xoloitzcuintle, el cual ha sido estudiado por la genética, la biología molecular, la medicina veterinaria, la zootecnia, la historia, la arqueozoología, la etnohistoria y la lingüística; mostrándose cómo promover su estudio al interior de las diferentes asignaturas, dentro de proyectos estudiantiles. Esta propuesta es igualmente aplicable a numerosas especies latinoamericanas que, al ser domésticas y muchas veces de carácter regional, pueden ser empleadas en estos proyectos escolares, para ver a través de ellas aspectos biológicos, médicos, históricos, culturales, de aprovechamiento, siempre dentro de un marco de cotidianeidad para el alumno, promoviendo en él la certeza del valor de estos recursos asociados con lo natural y lo cultural.

\section{Palabras clave}

Latinoamérica, educación, biodiversidad, diversidad cultural, especies domésticas.

1. Colegio de San Ignacio de Loyola Vizcaínas, Vizcaínas 21, Colonia Centro, Delegación Cuauhtémoc, C.P. 06080, Ciudad de México, Distrito Federal, México.

2. Instituto de Investigaciones Antropológicas de la Universidad Nacional Autónoma de México, Circuito Exterior, Ciudad Universitaria, Delegación Coyoacán, C.P. 04510, Ciudad de México, Distrito Federal, México.raul_valadez@hotmail.com 


\section{Abstract}

Currently much of Latin American people are engaged in a scheme where we live globalization on one hand and on the other our lifestyles with traditions product of centuries of history in specific biogeographic spaces. In this sense, educational schemes have the greatest commitment in safeguarding our biological heritage (resources) and cultural (traditions) to maintain learner awareness that he is part of it. Within the current educational schemes, in which the teaching through skill development and interaction of various disciplines towards an inclusive and promotes critical thinking is essential to have the elements of study that help to understand the biological phenomenon, the historical and cultural simultaneously. As given to these needs, the use of school projects directed toward the study of domestic Latin American animals, plants and fungi, since each has a biological and cultural history integrated into various Latin American regions, many have been studied by various disciplines and are part of the reality of the inhabitants of these regions. A case applicable to Mexican classrooms is the Mexican hairless dog or xoloitzcuintle, which has been studied by genetics, molecular biology, veterinary medicine, animal husbandry, history, archaeozoology, ethnohistory and linguistics, showing as the promotion of study within the different subjects within student projects. This proposal is equally applicable to many Latin American species that being domestical and often regional in nature, can be used in these school projects, to see through them biological, medical, historical, cultural, harvesting,always within a part of everyday life for students, promoting him certain value of these resources associated with the natural and the cultural.

\section{Key words}

Latin America, education, biodiversity, cultural diversity, domestic species.

América Latina constituye uno de los focos del planeta en los cuales la diversidad biótica y cultural ${ }^{3}$ se manifiestan en todo su esplendor. Desde el punto de vista de nuestra realidad biológica, el territorio que va desde México hasta Brasil y Perú constituye el más grande espacio de biodiversidad ${ }^{4}$ en la Tierra (Alvarado, 2000) y la historia geológica y natural de todo el territorio entre la Península de Baja California y Tierra del Fuego, incluido el Caribe, es sumamente rica, con una historia independiente de la que se dio en el resto del mundo.

3. Tomando como el concepto de cultura al conjunto de valores, costumbres, creencias y prácticas que constituyen la forma de vida de un grupo humano específico (Eagleton, 2001).

4. Los autores manejan como biodiversidad a la diversidad de especies, del acervo genético de cada una y de ecosistemas, en el planeta (Valadez, Téllez y Alvarado, 2012).
Desde el punto de vista cultural la situación es muy similar. Las poblaciones humanas que entraron al continente americano y rebasaron el Estrecho de Bering conforme avanzaron hacia el sur quedaron separadas y aisladas del resto del mundo desde hace más de diez mil años (Hirst, 2013; Waters y Stafford. 2007). Esta circunstancia llevó a que sus esquemas de vida, su relación con el ambiente y el posterior desarrollo de la civilización se realizaran de manera independiente de lo que ocurrió en otros sitios, por ejemplo China o Medio Oriente, dando lugar así a manifestaciones culturales originales y únicas.

A pesar de los cambios provocados por la llegada de los europeos al continente americano a partir del siglo xvi, en gran parte de este territorio continuó un esquema en el que las personas estaban integradas al ambiente del cual obtenían lo necesario para vivir y por lo mismo sus esquemas culturales obedecían a tradiciones y formas de pensar regionales. Esto cambia por completo en las últimas décadas del siglo XX, con los fenómenos de neoliberalismo y globalización, ya que a través de ellos las grandes transnacionales se apropian de los recursos de vastas regiones del planeta sin ningún interés en preservar los legados naturales, y las vastas redes de comunicación masiva impulsan la homogeneidad en las formas de pensamiento, haciendo a un lado la diversidad cultural.

Sin duda este marco es el principal justificante de la etnobiología latinoamericana actual, pero desafortunadamente no siempre se tiene la noción de cómo transferir estas inquietudes, o los productos de las investigaciones realizadas, a las generaciones jóvenes (Valadez y Téllez, 2012), aspecto que, sin duda, es otro de sus compromisos fundamentales. De ello surge la pregunta: ¿Es posible impulsar la salvaguarda del acervo biológico y cultural en el salón de clase?

Durante mucho tiempo, dentro de los planes y programas de educación básica, en este caso la relacionada con las ciencias naturales, buscaban el conocimiento de los fenómenos biológicos de manera general, y no regional, y muchas veces el discurso se enfocaba hacia casos ajenos a Latinoamérica, por ejemplo mostrando cómo funciona un ecosistema con un caso de la sabana africana o bien ilustrando la relación hombre-naturaleza con ejemplos europeos, evitando, incluso de manera intencional, la referencia a formas de pensamiento, tradiciones o manejo de recursos regionales. Afortunadamente ahora se busca que el estudiante conozca y valore la diversidad biológica y cultural de la zona donde vive.

Hace algunos años la Unesco hizo la Declaración sobre la educación superior en el siglo XXI (Paul y Elder, 2003), que indica: 
Las instituciones educativas deben formar a los estudiantes para que se conviertan en ciudadanos bien informados y profundamente motivados, provistos de un sentido crítico y capaces de analizar los problemas de la sociedad, buscar soluciones, aplicarlas y asumir responsabilidades sociales.

Propuesta que al paso de los años ha sido reforzada de acuerdo con lo establecido por el mismo organismo en el Compromiso para una Educación para la Sostenibilidad, mediante el desarrollo de acciones educativas a realizar durante la Década de Educación para un Desarrollo Sostenible (2005-2014) (2005), mismas que promueven que la educación debe buscar la construcción de sociedades con equidad, contemplando tanto el desarrollo personal como los aspectos ambientales, socioambientales y culturales (Vega, Freitas, Álvarez y Fleuri, 2009).

Afortunadamente estas declaraciones han tenido respuesta. En México, por ejemplo, la Secretaría de Educación Pública (SEP), en el marco de la Reforma Integral de la Educación Básica (RIEB), pone en manos de los maestros y maestras los Programas de Estudio 2011 y un curso optativo dentro de la Licenciatura en Educación Primaria (2012) sobre "Educación Ambiental para la Sustentabilidad” (Secretaría de Educación Pública, 2012), dentro de los cuales se promueve que sea el docente el responsable de generar los ambientes propicios para el aprendizaje, presentando situaciones que despierten el interés de los estudiantes para involucrarlos en actividades que les permitan valorar a la ciencia como un proceso social en construcción permanente (Rodríguez, 2011; Valadez y Téllez, 2012) que busca el mejoramiento social y ambiental.

En estos programas se solicita el tratamiento de los temas desde contextos cercanos, familiares e interesantes, en donde a partir de constantes preguntas:

- Se desencadene la curiosidad y la necesidad de investigar.

- Se organice la información obtenida.

- Se estimule la experimentación así como el trabajo colaborativo.

- Se haga uso de las Tecnologías de la información y comunicación, así como diversos recursos del medio, todo con el fin de generar experiencias (aprendizajes significativos) en los estudiantes para enriquecerlos y en su caso reorientarlos, creando aplicaciones únicas con lo aprendido, es decir, fomentando la creatividad.
Para el caso de la enseñanza de Ciencias 1 (biología), las competencias que deben desarrollarse a lo largo de $1^{\circ}$ de secundaria en adolescentes de entre 12 y 14 años de edad son (Rodríguez, 2011):

1. Comprensión de fenómenos y procesos naturales desde la perspectiva científica. En este proceso los alumnos plantean preguntas y buscan respuestas sobre diversos fenómenos y procesos naturales para fortalecer su comprensión del mundo. Igualmente, podrán diseñar y realizar proyectos, experimentos e investigaciones, así como argumentar, utilizando términos científicos de manera adecuada y fuentes de información confiables, en diversos contextos y situaciones para desarrollar nuevos conocimientos.

2. Toma de decisiones informadas para el cuidado del ambiente y la promoción de la salud orientadas a la cultura de la prevención. Los alumnos deben participar en acciones que promuevan el consumo responsable de los componentes naturales del ambiente y colaboren de manera informada en la promoción de la salud; analicen, evalúen y argumenten respecto a las alternativas planteadas sobre situaciones problemáticas socialmente relevantes y desafiantes desde el punto de vista cognitivo. Asimismo, que tomen decisiones y realicen acciones para favorecer la conformación de una ciudadanía respetuosa, participativa y solidaria.

3. Comprensión de los alcances y limitaciones de la ciencia y del desarrollo tecnológico en diversos contextos. Implica estimular en los alumnos la valoración crítica de las repercusiones de la ciencia y la tecnología en el ambiente natural, social y cultural; asimismo, relacionar los conocimientos científicos con los de otras disciplinas para explicar los fenómenos y procesos naturales, y aplicarlos en contextos y situaciones de relevancia social y ambiental.

4. Estrategias para el desarrollo de proyectos de investigación en el aula de educación básica (secundaria) en México. Dentro del desarrollo curricular de la escuela básica (secundaria) se solicita a los docentes impulsar el trabajo a través de proyectos en donde se trabaje de manera interdisciplinaria, utilizando como objetivos motivacionales temas de interés de los estudiantes (Exley y Dennise, 2007). 
Dentro de esta propuesta es fundamental que el docente conozca y maneje su área disciplinar, posea flexibilidad de pensamiento, disponibilidad para el trabajo colaborativo, que pueda integrar y revalorar conocimientos dentro del aula y capacidad para acompañar y dar seguimiento a los alumnos a lo largo de los proyectos (Pérez-Urria, 2010; Vega et al., 2009; Vicario, Smith, Gutiérrez-Zorrilla e Insausti, 2007).

\section{Objetivos}

A partir de lo planteado surge el objetivo del presente artículo: presentar una ruta de trabajo que permita cubrir la meta educativa, pero de tal forma que se enseñe y se aprenda con referentes propios de cada país o región biocultural, ${ }^{5}$ de tal modo que dentro del proceso se adquiera(n):

1. Los conocimientos biológicos que exige el plan curricular.

2. El valor del patrimonio biótico para esa región o país.

3. El saber tradicional que se tiene en dicho país o región respecto de tal o cual tema biológico.

4. Una visión de cómo se da la interacción entre el conocimiento biológico y el proveniente de otras disciplinas, los cuales han abordado al mismo objeto de estudio, por ejemplo información relacionada con su historia o su valor económico.

5. Las habilidades involucradas con el desarrollo de un proyecto escolar que permita reunir y analizar toda esta información con un solo propósito.

\section{Materiales y métodos}

- Si revisamos con detalle qué parte del entorno humano se encuentra en frontera con lo natural, de forma tal que se pueda considerar como objeto de estudio que cubre todos los objetivos arriba señalados, sin duda una alternativa son los organismos domésticos, ya que todos:

- Son especies cuya biología se conoce con gran detalle.

- Son conocidos por la gente, pues forman parte de su entorno y en muchas ocasiones están involucrados en su manejo y uso, es decir, forman parte de su vida cotidiana.

5. Territorio con características particulares en lo biológico y lo cultural (Torey, 2010).
- Su historia natural y su evolución biológica está incluida en regiones biogeográficas que forman parte de cada país y por tanto son bien conocidas por alumnos y profesores.

- Su interacción con el ser humano es una realidad, a veces desde tiempos muy antiguos, lo que permite vincularlos con la historia de tal o cual región o cultura.

- Su evolución hacia la condición doméstica sin duda ha sido objeto de estudio por la academia correspondiente, tanto a nivel biológico como cultural.

- Su larga historia de vinculación con el hombre ha derivado en un acervo de conocimiento tradicional.

- Han sido bien estudiados de manera formal por diversas disciplinas además de la biología, por ejemplo la medicina, la zootecnia, la agronomía, muchas veces para corroborar si el conocimiento tradicional es correcto.

- Están involucrados en cadenas productivas.

- Poseen esquemas de interacción hacia los ambientes naturales muy diversos, lo que les hace muy valiosos para ver aspectos relacionados con la conservación, protección a especies, equilibrio ambiental, etc.

- Comparten gran número de interacciones para con las poblaciones humanas, desde uso como mascota u ornato hasta lo industrial.

La historia de aislamiento que vivieron las poblaciones latinoamericanas desde hace más de diez mil años llevó a que en cada territorio se dieran procesos dentro de los cuales determinados animales, plantas u hongos se beneficiaron de la presencia humana hasta dar origen a especies que podían cubrir su ciclo de vida completo en estos ámbitos perturbados, especies domésticas o protodomésticas ${ }^{6}$ (Baker, 1968; Crockford, 2006; McClung y Zurita, 2001; Valadez, 2003; 2009; Valadez, Moreno y Gómez, 2011).

6. Damos la categoría de "protodomésticas" a aquellas especies o poblaciones cuyo esquema de vida se realiza dentro del ámbito humano, aprovechando los beneficios que obtiene, pero sin que el hombre participe activamente en el proceso. En la actualidad se considera que muchos de los animales domésticos de la antigüedad entraron a esta categoría como resultado de un proceso adaptativo, dentro del cual los individuos más tolerantes y flexibles se beneficiaban al vivir dentro de un territorio perturbado que no todos soportaban y en la medida que esto les eliminaba competencia y depredación, daba pie a un proceso de selección natural que llevaba a la constitución de poblaciones capaces de interactuar con lo humano, es decir, "casi domésticas", sin que Homo sapiens tuviera conciencia de lo ocurrido (Crockford, 2006; Valadez, 2009). Este mismo principio, ajustado a su marco biológico, permite asignarle el término de protodoméstico, por ejemplo al cuitlacoche (término náhuatl para denominar en México al hongo del maíz, Ustilago maydis) (Valadez et al., 2011) y sin duda es aplicable a numerosas plantas, hongos y animales. 
Este proceso, aunque ignorado en sus inicios, posteriormente fue apoyado por Homo sapiens en la medida que se beneficiaba de los productos (organismos cercanos al ámbito humano, más dóciles, más resistentes, capaces de soportar la alteración). En varias ocasiones este fenómeno incluía especies endémicas, aspecto que le da al proceso un especial valor biológico; en otras ocasiones se trató de organismos de distribución amplia pero que solo pasaron a la categoría de domésticos en ciertos lugares, muchas veces por los intereses humanos, situación que promueve su importancia como tema de estudio antropológico (tabla 1).

\begin{tabular}{|c|c|c|}
\hline Organismo & Nombre científico & $\begin{array}{l}\text { Espacio latinoamericano asociado a su } \\
\text { domesticación e historia }\end{array}$ \\
\hline Cobayo & Cavia porcellus & Zona andina (Perú, Ecuador) \\
\hline Perro & Canis lupus familiaris & Todo el continente \\
\hline Llama & Lama glama & Zona andina, (Colombia a Argentina) \\
\hline Pato criollo & Cairina moschata domestica & El Chaco (Bolivia, Paraguay, Argentina) \\
\hline Guajolote & Meleagris gallopavo & México \\
\hline Guacamaya roja & Ara macao & México \\
\hline Centzontle & Mimus polyglottus & México \\
\hline Abeja sin aguijón & Melipona beecheii, M. yucatanica & Sureste de México y Centroamérica \\
\hline Maíz & Zea mays & Todo el continente \\
\hline Frijol & Phaseolus vulgaris & México, Centroamérica, Antillas, zona andina \\
\hline Naranjilla & Solanum quitoense & Colombia, Ecuador y Perú \\
\hline Papa & Solanum tuberosum & Zona andina \\
\hline Batata & Ipomea batata & México a zona andina, Antillas \\
\hline Cacahuate & Arachis hypogaea & Noroeste de Argentina, sur de Bolivia \\
\hline Yerba mate & Ilexparaguariencis & Paraguay, Argentina, Uruguay \\
\hline Aguacate & Persea americana & México \\
\hline Achogcha & Cyclanthera pedata & América central, Colombia, Ecuador y Perú \\
\hline Calabaza, zapallo & Cucúrbita pepo y C. maxima & México, América Central \\
\hline Chile & Capsicum annum & México \\
\hline Jitomate & Lycopersicum esculentum & México \\
\hline Capulín & Prunus salicifolia & México \\
\hline Cupa & Cissus gongylodes & Brasil, Paraguay, Argentina \\
\hline Guayaba & Psidium guajava & México \\
\hline Mashua & Tropaeolum tuberosum & Zona andina \\
\hline Melloco & Ullucus tuberosus & Venezuela, zona andina, Argentina \\
\hline Oca & Oxalis tuberosa & Zona andina \\
\hline Quinoa & Chaenopodium quinoa & Zona andina, desde Colombia hasta Chile y Argentina \\
\hline Zanahoria blanca & Arracacia xanthorriza & Zona andina, desde Venezuela hasta Bolivia \\
\hline Algodón & Gossypium herbaceum & México \\
\hline Hongo del maíz & Ustilago maydis & México \\
\hline
\end{tabular}

Tabla 1. Treinta especies de animales, plantas y hongos domésticos y cultivados

Nota: La tabla presenta datos generales de treinta especies de animales, plantas y hongos domésticos y cultivados, cuya historia biológica y cultural les liga con los pueblos latinoamericanos desde tiempos muy remotos. La lista, aunque amplia, es solo un pequeño ejemplo de organismos que pueden ser estudiados en proyectos escolares por diversas asignaturas de modo simultáneo. 
Como se muestra en la tabla1, los ejemplos son numerosos, lo mismo que las regiones en donde se dio el proceso, lo que permite utilizarlos en prácticamente cualquier lugar de Latinoamérica. Solo es cuestión de decidir cuáles serían los más adecuados para cubrir los objetivos escolares y disponer de argumentos adecuados para la construcción de un proyecto (LaCueva, 2000).

Obviamente el espacio de este artículo es insuficiente para abordar cada caso con detalle, por lo que para ilustrar la propuesta se empleará un solo organismo, el perro pelón mexicano o xoloitzcuintle, el cual ha sido objeto de estudio formal desde hace varias décadas, lo que permite disponer de un abanico de información alrededor de él (Drögermüller et al., 2008; Hernández, 2010; Leonard et al., 2002; Schnaas, 1974; Rosano-Hernández, 2002; Valadez y Mestre, 1998; 1999; 2007; Valadez, Téllez, Mestre, y González, 1998; Valadez, Leonard y Vilá, 2003; Valadez, Götz y Mendoza, 2010).

Desde hace varios años se determinó que el perro (Canis lupus familiaris) se derivó de lobos grises asiáticos (Leonard et al., 2002), sin embargo, es un hecho que entraron al continente americano casi tan temprano como el hombre mismo; lo que hace que la historia de este animal dentro de las culturas latinoamericanas sea sumamente rica e interesante.

Respecto del perro pelón en particular, esta raza es de origen americano, tal y como lo demuestra su mención en obras españolas de los siglos XVI (Hernández, 1959; Sahagún, 1979). Después de tres siglos de indiferencia, en el siglo XX fue redescubierto por criadores y diversas personas de tendencia nacionalista (al menos en México), impulsando su crianza (Valadez y Mestre, 1999; 2007). Además de su propia condición pelona, sin duda su carácter más destacable son sus camadas mixtas, esto es, con presencia de especímenes con la piel desnuda o cubierta de pelo, cuestión que trató de ser respondida y abordada a través de cruzas selectivas y sacrificio sistemático de los cachorros con pelo (Wright, 1960), hasta que se demostró que se trataba de una condición genética inherente a la raza y, por tanto, imposible de modificar (Schnaas, 1974; Valadez y Mestre, 1998). Otros países de Latinoamérica (Cuba, Ecuador, Perú, Bolivia, Paraguay y Argentina) poseen poblaciones de perros pelones, algunas reconocidas formalmente como descendientes de xoloitzcuintles, otras ubicadas más bien como razas equivalentes con su propia historia y acervo tradicional proveniente de tiempos pasados (Darwin, 1921; Guamán Poma de Ayala 1988; Mendoza, 2004; Valadez et al., 2010; Weiss, 2000).
Desde el punto de vista biológico, siempre ha sido objeto de atención su condición mixta (figura 1), por lo que se conoce con detalle su genética, incluso el gen del cual se derivó su condición de alopecia; su patrón de herencia, que se ajusta maravillosamente a los principios mendelianos; su histología y su adn, lo cual permite ubicar su posición dentro de la genealogía de los perros.

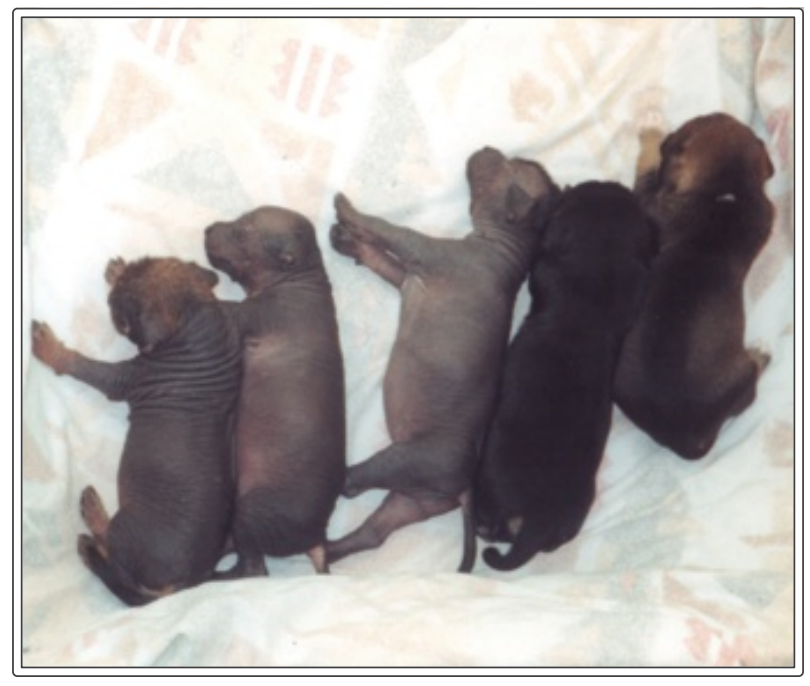

Figura 1. Camada de perros pelones mexicanos. Los tres cachorros del lado izquierdo son condición pelona y los dos de la derecha tienen el cuerpo cubierto de pelo. Este carácter, propio de todas las poblaciones de perros pelones del mundo, proviene de un solo gen, por lo que la forma como se da la herencia de este carácter obedece de modo ejemplar a los principios mendelianos. De esta forma, su genética, historia antigua y reciente, forma de vida y cuidados, le convierten en un buen ejemplo de animal doméstico apto para el desarrollo de proyectos escolares.

Como raza se considera un tipo primitivo, con pautas de comportamiento características, se conoce con detalle su manejo, su cuidado, su alimentación, sus usos. Debido a su condición mixta y a la presencia de tres tallas, es posible reconocer seis variedades en total.

Respecto de su historia, se dispone de información arqueológica para reconocer su presencia en asentamientos prehispánicos mexicanos desde hace dos mil años, sus patrones de dispersión, usos, simbolismo y nombres regionales.

A nivel médico, se le ha asociado con el tratamiento de reumas y otros problemas musculares y articulares y aunque esta idea parte de conceptos tradicionales, es médicamente correcto. 
Como perro al interior de las poblaciones humanas, las respuestas de la gente son enormemente dispersas, pues van desde su encumbramiento como símbolo nacional hasta su desprecio por su origen americano o su propia condición. Esto ha derivado en estudios sociológicos a fin de reconocer los orígenes de estas concepciones alrededor de él.

Incluso su condición pelona llevó en años pasados a que se hicieran estudios de cosmetología para reconocer como actuaban ciertos químicos sobre la piel desnuda.

Con base en esto su empleo dentro de los proyectos escolares es aplicable a diversas asignaturas, las cuales pueden compartir intereses, esquemas de trabajo y productos, favoreciendo así el pensamiento integrador y crítico, empleando un organismo que les es familiar o, al menos, que posee antecedentes culturales conocidos para los estudiantes.

\section{Resultados y discusión}

A continuación se mostrará cómo se estructuraría una secuencia didáctica dentro del curso de Ciencias 1, biología, de primero de secundaria (alumnos de 12 a 14 años) en México. Es importante señalar que la presentación de este esquema constituye solo un ejemplo, ya que uno de los objetivos a cubrir es que los estudiantes construyan su proyecto, quedando el docente como un orientador (Valadez, Téllez y Alvarado, 2012).

\section{El proyecto escolar en marcha \\ Ciencias 1. Biología.}

Unidad 1. La biodiversidad: resultado de la evolución. Tema: El valor de la biodiversidad.

\section{Aprendizajes esperados:}

- Se reconoce como parte de la biodiversidad al comparar sus características con las de otros seres vivos, e identifica la unidad y diversidad en relación con las funciones vitales.

- Representa la dinámica general de los ecosistemas considerando su participación en el intercambio de materia y energía en las redes alimentarias y en los ciclos del agua y del carbono.

- Argumenta la importancia de participar en el cuidado de la biodiversidad, con base en el reconocimiento de las principales causas que contribuyen a su pérdida y sus consecuencias.

\section{Duración:}

Un bimestre.

Inicio:

1. Presentar a los estudiantes fotografías en donde se observen diversas manifestaciones culturales, sociales, educativas, del objeto de estudio, en este caso, el xoloitzcuintle, y con ello promover una lluvia de ideas para indagar los conocimientos previos.

2. En el pizarrón dibujar cinco columnas (sin ponerles todavía un título) e ir anotándolas y agrupándolas de acuerdo a la siguiente clasificación: biología, geografía, historia, medicina y arte.

\section{Desarrollo:}

3. Poner el título a cada una de las columnas y preguntar a quién le interesa conocer más sobre $x$ tema e ir agrupando a los estudiantes de acuerdo con los intereses que expresen, así se integrarán los equipos de trabajo.

4. Se formarán cinco equipos de cuatro estudiantes; cada uno escogerá una función que desempeñará al interior del equipo, la cual irán cambiando a lo largo de la investigación, es decir, todos los miembros al final deberán haber desempeñado todas las funciones (Valadez et al., 2012):

Secretario: Integra la información que recolectan de diversas fuentes (libros, revistas, sitios de Internet, entrevistas, visitas, etc.).

Dibujante: Elaborará y extraerá de Internet; dibujos, esquemas, mapas, etc. Todas las imágenes que complementen el tema de investigación.

Coordinador: Organizará el trabajo, revisará que las actividades se cumplan en los tiempos establecidos, apoyando a los integrantes del equipo con alguna tarea en particular.

Moderador: Organizará el trabajo de exposición ante el grupo sobre los resultados de la investigación. Es importante que todos los integrantes del equipo participen en la exposición, pues todos conocen muy bien el tema de investigación. 
5. Ya determinado el tema y las funciones de cada uno de los integrantes, completarán el organizador "¿Qué sé, qué quiero saber, qué aprendí?" (SQA) (Ogle, 1986) para orientarlos en el desarrollo de su investigación.

\section{¿Qué sé?}

\section{¿Qué quiero saber?}

6. Ya que identificaron lo que quieren saber sobre el tema que escogieron, es importante orientarlos en el desarrollo de la construcción de su planeación, desde el inicio hasta el final, con la comunicación de los resultados a sus compañeros y a la comunidad en general.

7. Es importante que generen su hipótesis, planten el tiempo y las fuentes bibliográficas que consultarán para su investigación.
Nombre de la investigación: / Fecha de inicio: / Fecha de finalización: Objetivos o metas: / Actividades a realizar, tiempos y responsable:

8. Con base en el organizador sobre lo que se conoce y lo que quieren saber, apoyarlos en la construcción de las preguntas desencadenantes que dan inicio a la investigación.

- ¿Cómo los puedes distinguir de otros perros?

- ¿Por qué se dice que tienen camadas mixtas?

- ¿Qué cuidados se les deben proporcionar?

- ¿Cuántos y cuáles nombres tienen de acuerdo a las regiones del país?

- ¿Cómo eran utilizados en el México prehispánico?

- ¿Cómo son utilizados en la actualidad?

- ¿Qué tradiciones hay en torno a él?

- ¿Cuál es su importancia dentro del arte?

9. Para esto es muy importante la realización de las fichas bibliográficas, a partir de las cuales irán construyendo el cuerpo de la investigación. A continuación se presenta una sugerencia (figura 2):

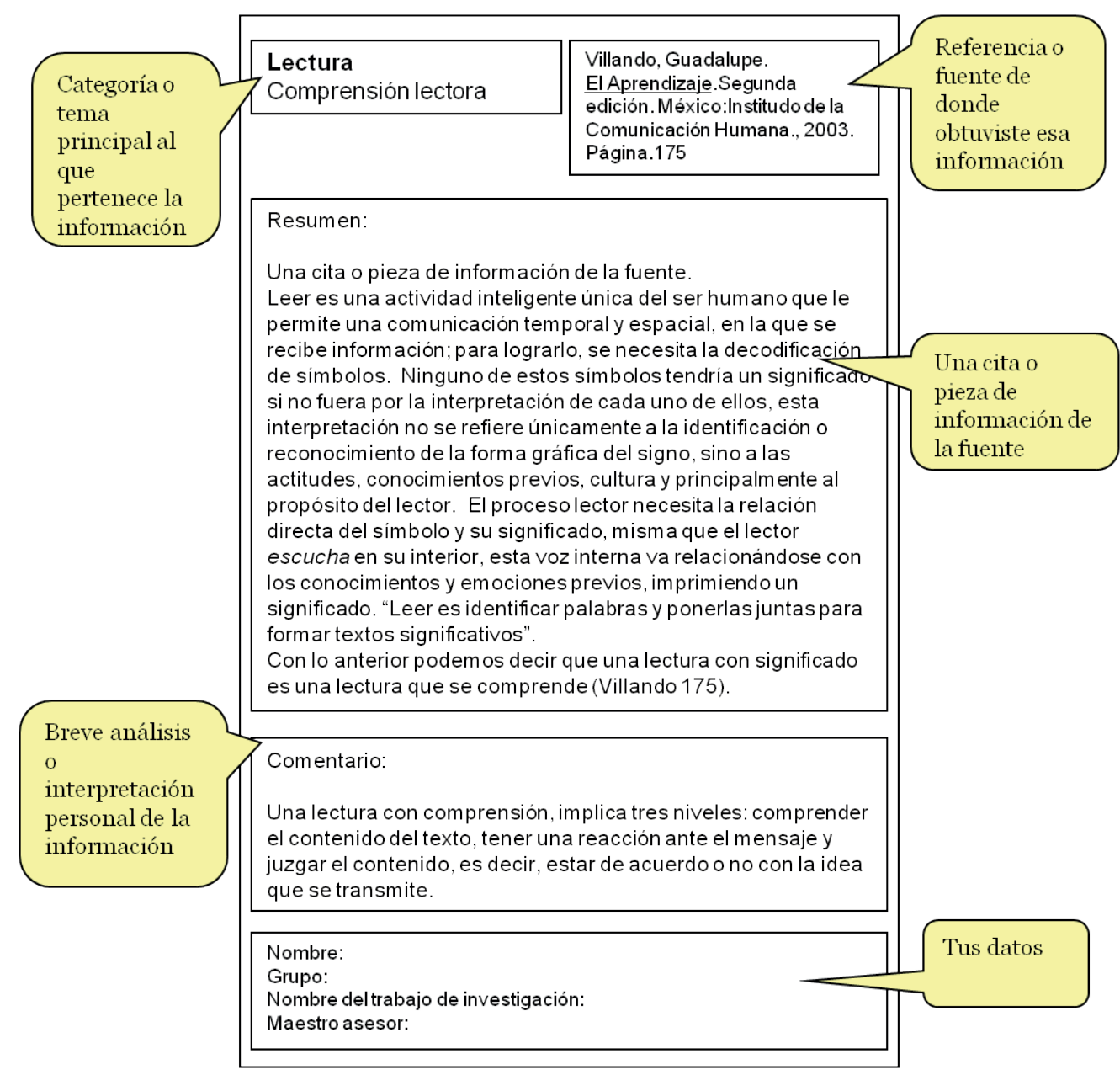

Figura 2. Ejemplo de ficha bibliográfica con los rubros que deben ser considerados 
10. Es de suma importancia el seguimiento a lo largo de las actividades que realizarán en cada una de las fases del desarrollo del proyecto (Pantoja y Covarrubias, 2013).

11. Es indispensable fomentar una comunicación directa y constante entre los estudiantes y entre ellos y el docente.

12. Para finalizar se les orientará en la elaboración de sus conclusiones basada en su investigación y experiencias, es decir, una explicación de los resultados. Para ello pueden partir de las siguientes preguntas:

- ¿Se cubrió el objetivo de la investigación?

- ¿La metodología utilizada fue la correcta? ¿Por qué?

- ¿Qué aprendimos a lo largo de la investigación?

- ¿A qué dificultades nos enfrentamos?

- ¿Qué acciones podemos implementar a partir del estudio del xoloitzcuintle?

- ¿Qué otras investigaciones podríamos generar a partir de esta?

\section{Cierre:}

Existen muchas formas de expresar las ideas y experiencias acumuladas a lo largo del proyecto, por lo que es muy importante que cada uno de los equipos comunique al resto de sus compañeros los resultados de su investigación, intercambien sus ideas y productos obtenidos. De esta forma todos los integrantes del grupo se enriquecerán en conocimientos y experiencias.

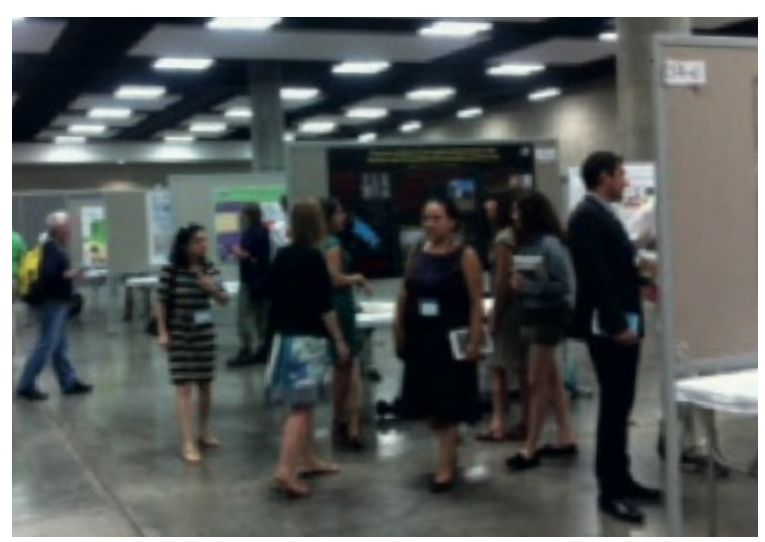

Figura 3. La comunicación de los resultados a la comunidad es una experiencia muy valiosa dentro del proceso de enseñanzaaprendizaje
Mientras se presentan los resultados, es necesario integrar, en un cuadro comparativo, lo más relevante de cada una de las investigaciones, para que al final del periodo tengan una visión global e integrada de la importancia del perro pelón en México.

Es recomendable que estas investigaciones se den a conocer a toda la comunidad a través de los medios disponibles (presentaciones en computadora, pósteres, conferencias, videos, etc.) (figura 3).

\section{Evaluación:}

La evaluación es considerada como una actividad que apoya el trabajo de los estudiantes de manera objetiva, un espacio en donde se hacen "altos" para analizar las acciones, los productos $y$, si es necesario, redirigir el plan de trabajo.

Una de las vías (aunque no la única) para lograr evaluar con mayor calidad, cualitativa y procesual, es el empleo de protocolos de evaluación o rúbricas (tabla 2). Estas favorecen una evaluación más sistematiza por parte del docente, además de considerarse como una herramienta de extraordinario valor para el desarrollo de competencias de autoevaluación y evaluación entre pares, así como el contribuir a un mayor entendimiento del propio proceso de aprendizaje, llevando a los estudiantes a una mayor autonomía y autorregulación (Stevens y Levi, 2005). Para ello es recomendable realizar evaluaciones en cada una de las fases del proyecto.

El abordar los contenidos curriculares a partir de la construcción de proyectos motiva a los estudiantes a actuar como individuos inquietos y críticos que se informan, proponiendo soluciones de manera colaborativa que generen más conflictos cognitivos, llevándolos a proponer la elaboración de nuevos proyectos (Díaz Barriga, 2005). Una acción imprescindible del docente es estar muy pendiente del trabajo que realizan los estudiantes y dar seguimiento de manera personal, por equipo y grupal, favoreciendo la comunicación directa, honesta y respetuosa entre los integrantes del equipo y hacia el medio en el que están desarrollando sus proyectos. De la misma manera es, importante que el docente cuente con información actualizada sobre el tema, o los temas a trabajar. 


\begin{tabular}{|c|c|c|c|c|}
\hline & 10 & 8 & 6 & 0 \\
\hline$\frac{\frac{0}{0}}{\frac{0}{\frac{1}{2}}}$ & $\begin{array}{l}\text { Trae el material necesario a } \\
\text { clase y siempre está listo para } \\
\text { trabajar. }\end{array}$ & $\begin{array}{l}\text { Casi siempre trae el material } \\
\text { necesario a clase y está listo para } \\
\text { trabajar. }\end{array}$ & $\begin{array}{l}\text { Casi siempre trae el material } \\
\text { necesario, pero algunas veces } \\
\text { necesita instalarse y se pone a } \\
\text { trabajar. }\end{array}$ & $\begin{array}{l}\text { A menudo olvida el material nece- } \\
\text { sario o no está listo para trabajar. }\end{array}$ \\
\hline \multirow[b]{2}{*}{ 올 } & 10 & 8 & 6 & 5 \\
\hline & $\begin{array}{l}\text { Nunca critica públicamente } \\
\text { el trabajo de otros. Siempre } \\
\text { tiene una actitud positiva } \\
\text { hacia el trabajo. }\end{array}$ & $\begin{array}{l}\text { Rara vez critica públicamente el tra- } \\
\text { bajo de otros. A menudo tiene una } \\
\text { actitud positiva hacia el trabajo. }\end{array}$ & $\begin{array}{l}\text { Ocasionalmente critica pública- } \\
\text { mente el trabajo de otros miem- } \\
\text { bros del grupo. Tiene una actitud } \\
\text { positiva hacia el trabajo. }\end{array}$ & $\begin{array}{l}\text { Con frecuencia critica en pública- } \\
\text { mente el trabajo de otros miembros } \\
\text { del grupo. A menudo tiene una acti- } \\
\text { tud negativa hacia el trabajo. }\end{array}$ \\
\hline \multirow[b]{2}{*}{ 을 } & 10 & 8 & 6 & 0 \\
\hline & $\begin{array}{l}\text { El trabajo refleja los mejores } \\
\text { esfuerzos del estudiante. }\end{array}$ & $\begin{array}{l}\text { El trabajo refleja un esfuerzo } \\
\text { grande por parte del estudiante. }\end{array}$ & $\begin{array}{l}\text { El trabajo refleja algo de esfuerzo } \\
\text { por parte del estudiante. }\end{array}$ & $\begin{array}{l}\text { El trabajo no refleja ningún } \\
\text { esfuerzo por parte del estudiante. }\end{array}$ \\
\hline \multirow{2}{*}{ 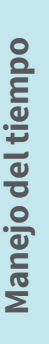 } & 20 & 15 & 10 & 5 \\
\hline & $\begin{array}{l}\text { Utiliza bien el tiempo durante } \\
\text { toda la investigación para ase- } \\
\text { gurar que las productos se } \\
\text { entregaran dentro de los pla- } \\
\text { zos marcados por el equipo. }\end{array}$ & $\begin{array}{l}\text { Utiliza bien el tiempo durante } \\
\text { toda la investigación pero algu- } \\
\text { nos de los productos no se entre- } \\
\text { gan dentro de los plazos marca- } \\
\text { dos por el equipo. }\end{array}$ & $\begin{array}{l}\text { Tendió a demorarse, pero siem- } \\
\text { pre entregó las productos dentro } \\
\text { de los plazos marcados por el } \\
\text { equipo. }\end{array}$ & $\begin{array}{l}\text { Rara vez tuvo los productos elabo- } \\
\text { rados dentro de los plazos marca- } \\
\text { dos por el equipo, dado lo cual se } \\
\text { ajustaron las fechas límites marca- } \\
\text { das por el equipo. }\end{array}$ \\
\hline \multirow{2}{*}{ 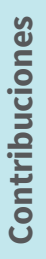 } & 20 & 15 & 10 & 5 \\
\hline & $\begin{array}{l}\text { Proporciona siempre ideas } \\
\text { útiles cuando participa en la } \\
\text { discusión del equipo. }\end{array}$ & $\begin{array}{l}\text { Por lo general proporciona ideas } \\
\text { útiles cuando participa en la dis- } \\
\text { cusión del equipo. }\end{array}$ & $\begin{array}{l}\text { Algunas veces proporciona ideas } \\
\text { útiles cuando participa en la dis- } \\
\text { cusión del equipo. }\end{array}$ & $\begin{array}{l}\text { Rara vez proporciona ideas útiles, } \\
\text { por lo regular se rehúsa a participa } \\
\text { en la discusión del equipo. }\end{array}$ \\
\hline \multirow{2}{*}{ 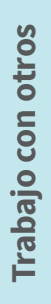 } & 15 & 10 & 5 & 2 \\
\hline & $\begin{array}{l}\text { Casi siempre escucha, com- } \\
\text { parte y apoya el esfuerzo de } \\
\text { otros. Trata de mantener la } \\
\text { unión de los miembros traba- } \\
\text { jando en equipo. }\end{array}$ & $\begin{array}{l}\text { Usualmente escucha, comparte } \\
\text { y apoya el esfuerzo de otros. No } \\
\text { causa "problemas" en el equipo. }\end{array}$ & $\begin{array}{l}\text { A veces escucha, comparte y } \\
\text { apoya el esfuerzo de otros, pero } \\
\text { algunas veces no es un buen } \\
\text { miembro del equipo. }\end{array}$ & $\begin{array}{l}\text { Raramente escucha, comparte y } \\
\text { apoya el esfuerzo de otros. Fre- } \\
\text { cuentemente no es un buen miem- } \\
\text { bro del equipo. }\end{array}$ \\
\hline \multirow{2}{*}{ 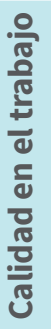 } & 10 & 8 & 6 & 2 \\
\hline & $\begin{array}{l}\text { Proporciona trabajo de la más } \\
\text { alta calidad. }\end{array}$ & Proporciona trabajo de calidad. & $\begin{array}{l}\text { Proporciona trabajo que, oca- } \\
\text { sionalmente, necesita ser com- } \\
\text { probado o rehecho por otros } \\
\text { miembros del equipo para ase- } \\
\text { gurar su calidad. }\end{array}$ & $\begin{array}{l}\text { Proporciona trabajo que, por lo } \\
\text { general, necesita ser comprobado } \\
\text { o rehecho por otros miembros del } \\
\text { equipo para asegurar su calidad. }\end{array}$ \\
\hline
\end{tabular}

\section{Tabla 2. Rúbrica de trabajo colaborativo a lo largo del bimestre}

Nota: La rúbrica debe ser realizada por cada uno los integrantes del equipo. Con ella, cada miembro del mismo realizará un ejercicio de autoevaluación y el docente evaluará al equipo. 


\section{El organismo doméstico: una realidad etnobiológica, cultural y educativa al alcance de todos}

Así como se indicó que la propuesta ofrecida constituía solo un ejemplo, así el organismo empleado es uno de muchísimas opciones disponibles, pues las especies domésticas, protodomésticas y cultivadas constituyen todo un universo en cantidad y diversidad que se encuentra junto o dentro de los territorios humanos, desde el campo de cultivo hasta el mercado, desde la maceta colocada en el patio de una casa hasta el área protegida más próxima.

Los procesos adaptativos que han llevado a estos seres vivos a convertir el entorno humano en su territorio es definitivamente el más interesante tema a considerar a nivel biológico, pues representa procesos evolutivos al alcance de uno, que pueden verse en el jardín más próximo. ¿Cuántas veces hemos enseñado evolución insistiendo en que todo debemos verlo con ayuda de la imaginación, argumentando que se trata de procesos lentos, antiguos, imposibles de ver en el presente? Bajo las nuevas teorías esto ha cambiado radicalmente y por lo mismo es posible verlo y recrearlo con cualquier especie doméstica o cultivada (Valadez, 2009; Valadez et al., 2011) (figura 3).
Para estos caso es igualmente valiosa e interesante la propuesta de cómo se ha dado la interacción de ambiente natural, el organismo y la población humana, dentro de un cierto territorio, para con una cultura determinada, o cómo es que se conceptualiza a una misma planta, hongo o animal, en diferentes regiones.

La riqueza de tradiciones alrededor de estos organismos, su cercanía a nosotros y el uso real y potencial lleva a que sean objeto de estudio continuo y a su revaloración, por lo que es relativamente sencillo hacerse de un buen acervo de publicaciones, a veces provenientes de entidades académicas que uno no se imagina o por grupos de personas que los estudian, protegen y valoran como parte de sus metas personales o profesionales (Anónimo a al d; Augstburger et al., 2000; Bonavia, 1993; Escobar, 2012; Flores, 2009; Gilbert, 2013; Hurtado, 2013; Jímenez, 2005; Morales, 2007; Pagán, 2005; Tagle y Planella, 2002; Tapia, 2013; Valadez et al., 2011; Vimos, Nieto y Rivera, 2013). Por último, dentro de este marco de ideas y alternativas, igualmente podemos impulsar proyectos escolares enfocados al estudio de una especie en particular o incluso, por ejemplo, la integración de la fauna o flora doméstica que existe en una determinada zona o país.

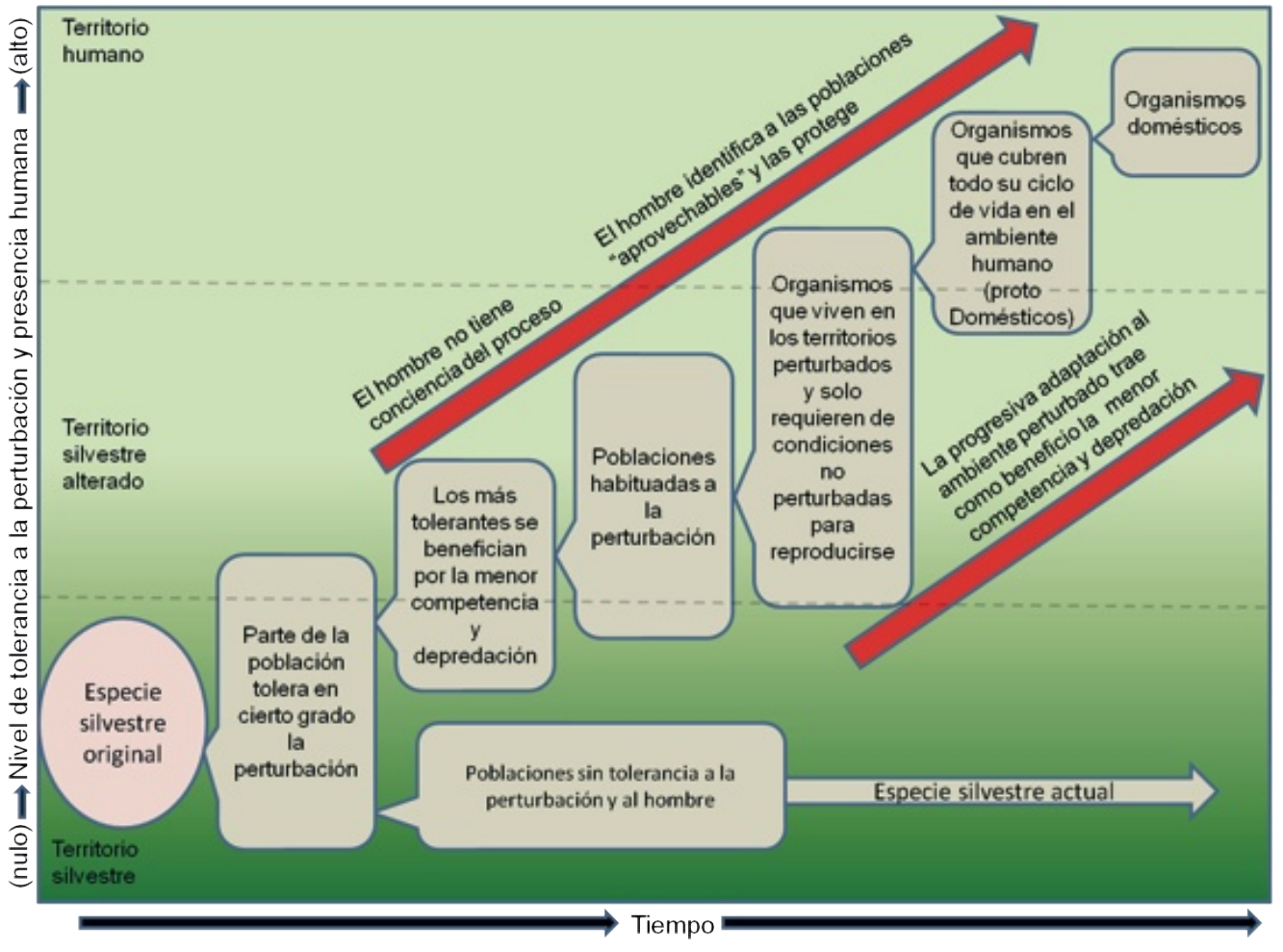

Figura 4. Modelo de evolución del organismo silvestre al doméstico a partir de la progresiva adaptación de una especie a los espacios perturbados por el hombre. Este proceso existe en la actualidad en todo espacio en el que el hombre altere o modifique el territorio, lo que permite verlo en diversos lugares, por ejemplo en las grandes ciudades (Valadez 2009). 
Los organismos domésticos han sido parte de la historia humana desde hace milenios y su cercanía para con el hombre, un puente real hacia la naturaleza, pero debido a que en la actualidad la expresión "especie doméstica" involucra producción de materia prima, tecnología o destrucción de ambientes naturales, lo usual es que se denigre su existencia, bien por los biólogos, que les consideran una plaga, o por antropólogos e historiadores, que simplemente les hacen a un lado en sus estudios. En este artículo vemos como gracias a la etnobiología latinoamericana podemos revalorarlos, mostrando su potencial cultural, biológico y educativo y de esta forma incluirlos dentro de una triada (etnobiología-educación-especie doméstica) a través de la cual se promueve en los jóvenes:

- Las actitudes responsables que se deben tener hacia la vida, en este caso un organismo doméstico.

- La concientización de que el organismo doméstico es parte de la biodiversidad, por tanto que cualquier especie merece nuestro apoyo y protección.

- Que el organismo doméstico forma parte de un conjunto de tradiciones que nos proporcionan identidad cultural.

- La valorización de nuestras riquezas biológicas y culturales.

- La necesidad de proteger estos acervos e impulsar un aprovechamiento sostenible.

\section{Conclusiones}

El trabajar a partir de la construcción de proyectos requiere de mucha planeación por parte de los docentes y tener muy claro el objetivo que se persigue, ya que su papel dentro de esta actividad es el de motivar, mediar y orientar las acciones que se realizan, a través de cuestionamientos que los lleven a la construcción del pensamiento crítico y reflexivo y a propiciar el trabajo colaborativo para conducir a los estudiantes a la independencia académica, primer paso dentro de la creación de individuos con libertad de pensamiento.

En el ejemplo mostrado hemos visto cómo, a través de esta propuesta educativa, es posible incluir a la etnobiología dentro de la educación formal, aspecto que igualmente requiere de colaboración y apertura (implícita o explícita) entre investigador y profesor. Para los primeros es fundamental no perder de vista la necesidad de disponer de estrategias que permitan hacer llegar los resultados de las investigaciones a las aulas, pues es ahí donde las nuevas generaciones reflexionan y deciden el tipo de mundo que impulsarán; y para los segundos, el acercarse a los diversos eventos académicos, así como entrar en contacto con investigadores con el objetivo de poder acceder a mucha de la información publicada, procesarla y transformarla en cuestionamientos que sean la base para la generación de nuevos proyectos.

\section{Referencias}

Anónimo (a). (Agosto 10, 2013). Cultivo de la batata, camote, boniato o papa dulce (Ipomea batatas). Extraído el 10 de agosto, 2013, de http://www.botanical-online.com/boniato_ipomoea_batatas_cultivo.htm

Anónimo (b). (Agosto 12, 2013). La mashua. Tubérculo de los Andes. Tratamiento natural próstata. En Hora buena, para que todas tus horas sean buenas. Extraído el 12 de agosto, 2013 de http://horabuena.blogspot.mx/2012/05/la-mashua-tuberculo-de-los-andes.html

Anónimo (c). (Agosto 12, 2013). Cyclanthera pedata. Extraído el 12 de agosto, 2013, de http://es.wikipedia.org/wiki/Cyclanthera_pedata

Anónimo (d). (Agosto 12, 2013). Oca (Oxalis tuberosa). Extraído el 12 de agosto, 2013, de http://www.peruecologico.com.pe/flo_oca_1.htm

Alvarado, A. (2000). Campamento Biofilia. La biodiversidad. México: Sociedad Mexicana para la Divulgación de la Ciencia y Técnica, Secretaría del medio Ambiente, Recursos Naturales y Pesca.

Augstburger, F., Berger, J., Censkowsky, U., Heid, P., Milz, J. y Streit, Ch. (2000). Maní (cacahuete). Agricultura orgánica en el trópico y subtrópico [Versión electrónica]. Alemania: Asociación Naturland.

Baker, H. G. (1968). Las plantas y la civilización. México: Serie Fundamentos de Botánica. Herrero Hermanos Sucesores.

Bonavia, D. (1993). La papa: apuntes sobre sus orígenes y su domesticación. Journal de la Société des Américanistes, 79, 173-187.

Crockford, S. (2006). Rhytms of Life. Thyroid Hormona \& the Origin of Species. Canada: Trafford Victoria.

Darwin, Ch. (1921). Diario de un naturalista alrededor del Mundo (Tomos I y II). Madrid: Editorial Calpe.

Díaz Barriga, F. (2005). Enseñanza situada: vínculo entre la escuela y la vida (Cap. 3, El aprendizaje basado en problemas y el método de casos). México: McGraw Hill. 
Drögermüller, C., Karlsson, E., Hitönen, M., Perloski, M., Dolf, G., Sainio, K. et al. (2008). A Mutation in Hairless Dogs Implicates FOXI3 in Ectodermal Development. Science, 321,1462.

Eagleton, E. (2001). La idea de cultura. Barcelona: Editorial Paidós.

Escobar, N. (2012). Estudio microbiológico y evaluación de la actividad antibacterial del aceite de semilla de Solanum quitoense (lulo), variedad la selva (Tesis para optar al título de Licenciatura en Química industrial). Escuela de Quimica, Facultad de Tecnología, Universidad Tecnológica de Pereira, Pereira, Colombia.

Exley, K. y Dennise R. (2007). Enseñanza en pequeños grupos en educación superior. Madrid: Narcea.

Flores, F. (2009). Cissus gongylodes: caracterização farmacognóstica e investigação de aspectos preliminares da seguridade da utilização de extratos aquosos das folhas e caules (Dissertação para obtenção do título de Mestrado, Programa de Pós-Graduação em Ciências Farmacêuticas). Área de Pesquisa e Desenvolvimento de Fármacos e Medicamentos, da Faculdade de Ciências Farmacêuticas, Universidade Estadual Paulista, Campus de Araraquara, Brasil.

Gilbert, P. (Agosto 10, 2013). Yerba mate y Ka'a he'ê. Extraído el 10 de agosto, 2013, de http://archivo. abc.com.

Guamán Poma de Ayala, F. (1988). El Primer Nueva Corónica y Buen Gobierno (2a ed.). México: Editorial Siglo XXI.

Hernández, F. (1959). Obras completas (Tomo III, tratado quinto, Historia natural de las cosas de la Nueva España). México: Universidad Nacional Autónoma de México.

Hernández, M. A. (2010). La construcción social del xoloitzcuintle moderno. En A. Moreno, M. T. Pulido, R. Mariaca, R. Valadez, P. Mejía y Gutierrez, T. (Eds.), Sistemas biocognitivos tradicionales. Paradigmas en la conservación biológica y el fortalecimiento cultural (pp. 53-66). México: Asociación Etnobiológica Mexicana A.C., Global Diversity Foundation, Universidad Autónoma del Estado de Hidalgo, El Colegio de la Frontera Sur, Sociedad Latinoamericana de Etnobiología.

Hirst, K. (Agosto 8, 2013). The pre-history of Clovis. Extraído el 08 de agosto, 2013, de http://archaeology.about.com
Hurtado, J. (Agosto 8, 2013). Achira, Canna indica. Animales y Vegetales de Perú. Extraído el 08 de agosto, 2013, de http://animalesyplantasdeperu

Jímenez, F. (2005). Características nutricionales de la arracacha (Arracacia xanthorrhiza) y sus perspectivas en la alimentación. Extraído el 12 de agosto, 2012, de http://www.faviolajimenez.com/wp-content/ uploads/2012/05/arracacha

LaCueva, A. (2000). La enseñanza por proyectos ¿Mito o reto? Revista Iberoamerica de Educación: Educación Ambiental y Formación: Proyectos y Experiencias, 16. Extraído el 11 de Agosto, 2013, de http://www.rieoei. org/oeivirt/rie16a09.htm

Leonard, J. A., Wayne, R. K., Wheeler, J., Valadez, R., Guillén, S. y Vilá, C. (2002). Ancient dna evidence for Old World origin of New World dogs. Science, 298, 1613-1616.

McClung E. y Zurita, J. (2001). Las primeras sociedades sedentarias. En L. Manzanilla y L. López, (Coords.), Historia Antigua de México (Vol. I) (2a edición) (pp. 255-295). México: Instituto Nacional de Antropología e Historia, Instituto de Investigaciones Antropológicas, Editorial Porrúa.

Mendoza, V. (2004). El perro en las sociedades andinas del pasado: un aporte arqueozoológico (Del Formativo al Inkario. Altiplano norte de Bolivia) (Tesis para optar al título de Licenciatura en Arqueología). Universidad Mayor de San Andrés, La Paz, Bolivia.

Morales, F. (2007). Sociedades precolombinas asociadas a la domesticación y cultivo de la papa (Solanum tuberosum) en Sudamérica [Versión electrónica]. Revista Latinoamericana de la Papa, 14(1), 1-9.

Ogle, D. (1986). K-W-L: A teaching model that develops active reading of expository text. The Reading Teacher, 39, 564-570.

Pagán, J. (2005). Estudio interpretativo de la cultura botánica de dos comunidades precolombinas antillanas: la Hueca y Punta Candelero, Puerto Rico (Tesis para optar al título de Doctorado en Antropología). Facultad de Filosofía y LetrasInstituto de Investigaciones Antropológicas, Universidad Nacional Autónoma de México, México.

Pantoja, C. y Covarrubias, P. (2013). La enseñanza de la biología en el bachillerato a partir del aprendizaje basado en problemas (abp). Perfiles Educativos, 35(139), 93-109. 
Paul R., Elder L. (2003). La mini-guía para el pensamiento crítico. Conceptos y herramientas. Fundación para el Pensamiento Crítico. Extraído el 9 de agosto, 2013, de www.criticalthinking.org

Pérez-Urria, C. (2010). Una propuesta de aprendizaje basado en problemas de fisiología vegetal. Estudio de casos. Mapas conceptuales. Infografías. Reduca (biología). Serie fisiología vegetal, 3(2), 18-31.

Rodríguez G. L. (2011). Programa de estudio 2011. Guía para el maestro. Educación básica secundaria. México: Secretaria de Educación Pública. Extraído el 8 de agosto, 2013 de http://www.excelduc.org.mx/ biblioteca/documentos/detalle.htm?id =34

Rosano-Hernández, M. C. (2002). Problemas dermatológicos comunes de los perros pelones mexicanos que viven en el sureste de México y la península de Yucatán. Revista de la Asociación Mexicana de Médicos Veterinarios Especialistas en Pequeñas Especies, 13(6), 236-241.

Sahagún, B. (1979). Códice Florentino (Libro XI). México: Secretaría de Gobernación.

Schnaas, G. (1974). El perro pelón; mito, fantasía y biología. Gaceta América de México, 108(6), 393-400.

Secretaría de Educación Pública (Diciembre 2, 2013). Educación ambiental para la sustentabilidad. Cursos optativos. Plan de Estudios 2012. Extraído el 2 de diciembre de http://www.dgespe.sep.gob.mx/ public/rc/programas/lepree/ educacion_ambiental_para_la_sustentabilidad_lepree.pdf

Stevens, D. D. y Levi, A. (2005). Introduction to rubrics: An assessment tool to save grading time, convey effective feedback, and promote student learning. Sterl ing, VA: Stylus Publishers. Extraído el 16 de Agosto, 2013, de http://www.introductiontorubrics.com

Tagle, M. B. y Planella, M. T. (2002). La quinoa en la zona central de Chile. Supervivencia de una tradición prehispánica. Santiago de Chile: Editorial IKU.

Tapia, M. (Agosto 12, 2013). La quinua. Historia, distribución geográfica, actual producción y usos. Revista Ambient@.Extraído el 12 de agosto, 2013, de http:// www.revistaambienta.es/WebAmbienta/marm/ Dinamicas/secciones/articulos/quinua.htm

Torey, S. (2010). Diversidad biocultural: un tesoro a medio explotar. Equitierra, Revista Rural Latinoamericana, 7, 32-37. Extraído el 25 de noviembre, 2013, de http:// www.fao.org/fileadmin/templates/olq/documents/ documents/Equitierra.pdf
Valadez, R. (2003). La domesticación animal (2a edición). México: Instituto de Investigaciones Antropológicas de la Universidad Nacional Autónoma de México.

Valadez, R. (2009). El fenómeno de la domesticación animal en los albores del siglo XXI. Revista de la Asociación Mexicana de Médicos Veterinarios Especialistas en Pequeñas Especies, 20(6), 136-148.

Valadez, R., Leonard, J. y Vilá, C. (2003). El origen del perro americano visto a través de la biología molecular. Revista de la Asociación Mexicana de Médicos Veterinarios Especialistas en Pequeñas Especies, 14(3), 73-82.

Valadez, R., Téllez R., Mestre, G. y González, C. (1998). Manejo y cuidados de los perros pelones mexicanos en el ámbito familiar. Revista de la Asociación Mexicana de Médicos Veterinarios Especialistas en Pequeñas Especies, 9(4), 141-146.

Valadez, R. y Mestre, G. (1998). El perro pelón, la realidad contra el mito y la fantasía. Revista de la Asociación Mexicana de Médicos Veterinarios Especialistas en Pequeñas Especies, 9(1), 21-26.

Valadez, R. y Mestre, G. (1999). Historia del xoloitzcuintle en México. México: Instituto de Investigaciones Antropológicas de la Universidad Nacional Autónoma de México, Museo Dolores Olmedo Patiño, Cámara de Diputados.

Valadez, R. y Mestre, G. (2007). El Xoloitzcuintle, del enigma al siglo XXI. México: Artenación editores, Instituto de Investigaciones Antropológicas de la Universidad Nacional Autónoma de México.

Valadez, R., Götz, Ch. y Mendoza, V. (2010). El perro pelón, su origen, su historia. México: Instituto de Investigaciones Antropológicas de la Universidad Nacional Autónoma de México, Universidad Autónoma de Yucatán.

Valadez, R., Moreno, A. y Gómez, G. (2011). Cujtlacochi (el cuitlacoche). México: Instituto de Investigaciones Antropológicas de la Universidad Nacional Autónoma de México.

Valadez, R. y Téllez R. (2012). Cosmos, corpus y praxis de la etnobiología mexicana aplicado en la educación básica. Etnobiología, 19(1), 52-59.

Valadez, R., Téllez R., Alvarado A. (2012). Vida, ambiente y salud. Ciencias 1 Biología. México: Editorial SM.

Vega, P., Freitas, M., Álvarez, P. y Fleuri, R. (2009). Educación Ambiental e Intercultural para la sostenibilidad: fundamentos y praxis. Utopía y Praxis Latinoamericana, 14(44), 25-38. 
Vicario, C., Smith, I, Gutiérrez-Zorrilla, J. M., Insausti, M., (2007). Aprendizaje basado en problemas. Una estrategia creativa para mejorar el aprendizaje en las titulaciones de biología y química. Proyecto de Innovación Educativa del Vicerrectorado de Innovación Docente de la UPUV/EHU, España: Universidad del País Vasco.

Vimos, C., Nieto, C. y Rivera, M. (Agosto 12, 2013). El melloco, características, técnicas de cultivo y potencial en Ecuador. Extraído el 12 de agosto, 2013, de http://archive.idrc.ca/library/document/096951/

Waters M. R., y Stafford, T. (2007). Redefining the Age of Clovis: Implications for the Peopling of the Americas [Versión electrónica]. Science 315, 1122-1126.

Weiss, P. (2000). El perro peruano sin pelo. Lima, Perú: Lluvia editores-Museo Nacional de Antropología, Arqueología e Historia.

Wright, N. (1960). El enigma del xoloitzcuintle. México: Instituto Nacional de Antropología e Historia. 